ISSN 2519-7398 (Versión electrónica)

DOI: http://dx.doi.org/10.21704/ac.v77i2.1203

(C) Universidad Nacional Agraria La Molina, Lima - Perú

\title{
Generación de energía eléctrica con un motor de combustión interna usando biodiesel de aceite de piñón (Jatropha curcas)
}

\author{
Electric power generation with an internal combustion engine using oil biodiesel pinion \\ (Jatropha curcas)
}

\author{
Fredy Omis Cáceres Guerrero'; José Luis Calle Maraví2
}

\begin{abstract}
Resumen
Dentro de los combustibles alternativos que cobran importancia por su valor ecológico se encuentran los biocombustibles, como el bioetanol, biometanol y el biodiesel. En este trabajo, se estudió de manera experimental el comportamiento de un motor diésel en una planta de generación de energía eléctrica empleando como combustible el biodiesel obtenido a partir de aceite del piñón (Jatropha curcas). Según los resultados obtenidos las mezclas que usaron del 20\% y $30 \%$ de biodiesel produjeron mayor potencia eléctrica, que la mezcla testigo (D-2) y la mezcla con el 10\% de biodiesel, pero se encontró diferencias significativas estadísticamente entre ellas. En cuanto al consumo horario de combustible se obtuvo el mismo comportamiento, es decir al incrementarse la potencia eléctrica, también se incrementó el consumo del combustible; sin embargo hubo diferencias estadísticamente significativas entre las mezclas. El consumo especifico fue mayor cuando la potencia eléctrica generada fue baja, es decir, el rendimiento del motor fue menor, pero a medida que aumentó la potencia eléctrica el consumo específico bajó a $0.5 \mathrm{Kg} / \mathrm{KW}$-h, manteniéndose constante a medida que la potencia eléctrica aumentó, lo que nos indicó que en este caso el rendimiento del motor de combustión interna fue alto, estadísticamente hubo diferencia significativa entre las mezclas, siendo mayor con la mezcla del 20\%. La opacidad generada por el motor de combustión interna de prueba tuvo una tendencia al incremento a medida que se incrementó la carga eléctrica, esto por el estado mecánico del motor, sobre todo por el sistema de inyección. Por otro lado, el torque generado en el motor de combustión interna al producirse la potencia eléctrica, tuvo un comportamiento lineal; sin embargo, se obtuvo diferencias significativas entre las mezclas.
\end{abstract}

Palabras Claves: biodiesel, potencia eléctrica, consumo de combustible, reducción de emisiones de gases contaminantes, Jatropha curcas.

\begin{abstract}
Within the alternative fuels that are becoming increasingly important for its ecological value are biofuels, such as bioethanol and biodiesel biomethanol. In this work, we study experimentally the behavior of a diesel engine in a plant of generation of electricity using biodiesel as fuel obtained from pinion oil (Jatropha curcas). According to the results, obtained mixtures that used $20 \%$ and $30 \%$ of biodiesel produced higher electrical power, warning that the mixture (D2 ) and the mixture with $10 \%$ biodiesel, but found statistically significant differences between them. About the hourly consumption of fuel was obtained the same behavior, which is to say in response to increased electrical power, it also increased the fuel consumption; however, there were statistically significant differences between mixtures. The specific consumption was higher when the electrical power generated was low, that is to say the performance of the engine was lower, but as you increase the electrical power consumption under specific to $0.5 \mathrm{Kg} / \mathrm{KW}$ - $\mathrm{h}$, remaining constant as the electrical power increase, that told us that in this case the performance of the internal combustion engine was high, statistically significant difference was found between the mixtures, being higher with the blend of $20 \%$. The opacity generated by the internal combustion engine test had a trend to increase as you increase the electrical load, this by the mechanical condition of the engine, especially by the injection system. On the other hand, the torque generated by the internal combustion engine to occur the electrical power, had a linear behavior; however obtain significant differences between the mixtures.
\end{abstract}

Keywords: biodiesel, electric power, fuel consumption, reduction of emissions of gaseous pollutants, Jatropha curcas.

\section{Introducción}

El problema del cambio climático, en especial el resultado del calentamiento global provocado por la acumulación de los gases de efecto invernadero, es un tema que en los últimos años ha tomado una gran relevancia, pues las variaciones se han acelerado crecientemente de manera anómala, a tal grado que pone en riesgo a la humanidad. Se señala como el principal responsable del calentamiento global al dióxido de carbono. 
Un biocombustible alternativo al diésel D-2 es el biodiesel, obtenido de productos vegetales oleaginosos, grasas animales o restos de aceites comestibles, y una de las principales ventajas de la utilización del biodiesel es la reducción de las emisiones de $\mathrm{CO}_{2}$, gracias al balance neutro de carbono en la combustión de biodiesel.

El fin supremo de las innovaciones y tecnologías modernas, es mejorar la calidad de vida de las personas en los lugares donde se aplica; a pesar de ello en nuestra selva y sierra del Perú existen lugares alejados de las grandes ciudades donde los pobladores viven todavía en situaciones muy precarias, sin las mínimas condiciones de servicios básicos.

El presente trabajo tiene como objetivo general, la generación de energía eléctrica a través de un motor de combustión interna y un generador de corriente alterna utilizando como combustible petróleo diésel D-2 y diferentes porcentajes de biodiesel a base de aceite de piñón (Jatropha curcas) en el mismo.

\section{Materiales y métodos}

Entre los materiales principales tenemos, la planta de ensayo y los medios de medición, para la toma de datos durante las pruebas, tales como: multímetro digital, cronómetro, pinza amperimétrica, tacómetro, beaker, balanza electrónica, opacímetro, etc. el motor diésel conectado a un generador, con una velocidad nominal de $1800 \mathrm{rpm}$, refrigerado por aire, teniendo un banco de 60 bombillas de 100 watts cada uno. (Figura 1 y Figura 2).

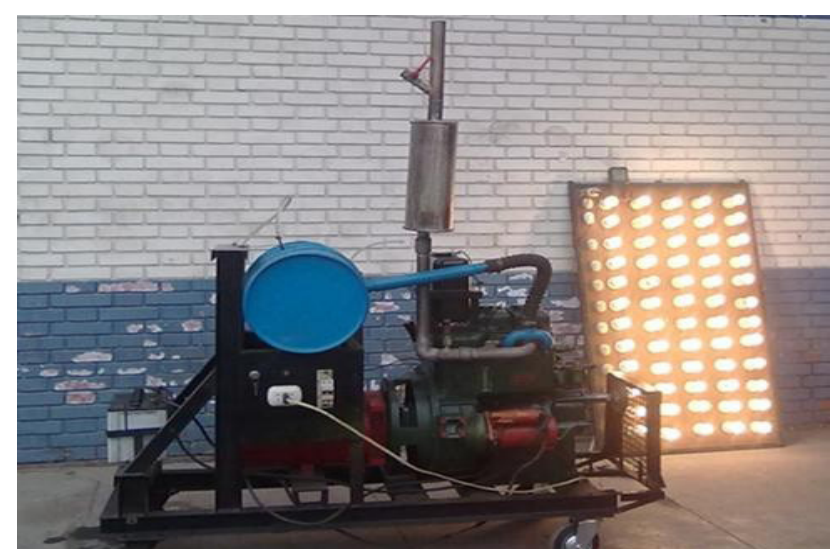

Figura 1. Planta de generación de energía eléctrica

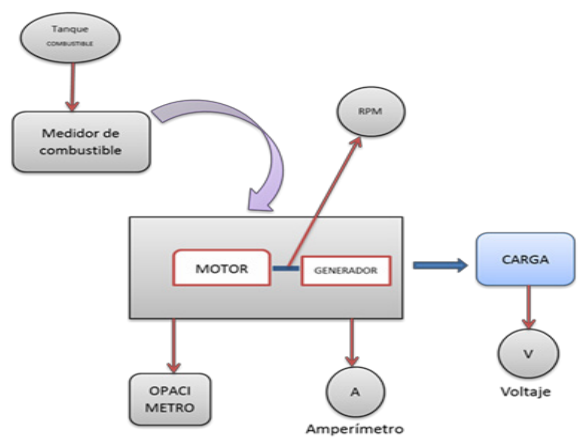

Figura 2. Esquema de la planta de prueba

\section{Tipos de combustible empleados:}

Actualmente los combustibles en el Perú por D.S. $\mathrm{N}^{\mathrm{o}}$ 021-2007-EM Reglamento de Comercialización de Biocombustibles, dispuso que a partir del año 2011 será de uso obligatorio el Diésel B5 $=5 \%$ de Biodiesel B100 + 95\% de Diésel 2; sin embargo, por gestiones del Instituto de Motores de Combustión Interna de la UNI, ante la Pampilla, se logró conseguir Diésel D-2 puro, los mismos que se usaron para las pruebas. Las mezclas que se utilizaron para las pruebas fueron: Diésel 2 puro $100 \%$ hidrocarburo, Diésel $2+10 \%$ de biodiesel, Diésel $2+20 \%$ de biodiesel y Diésel $2+30 \%$ de biodiesel.

\section{Métodos}

Proceso de extracción de aceite de los granos de Jatropha curcas

Entre los trabajos previos que se realizó en esta investigación, se encuentra la obtención de las semillas de Jatropha curcas, el mismo que procedió del norte del país; así se realizaron los pasos esquematizados en la Figura 3 y Figura 4, así como la Figura 3, 4, 5 y 6 del Anexo.

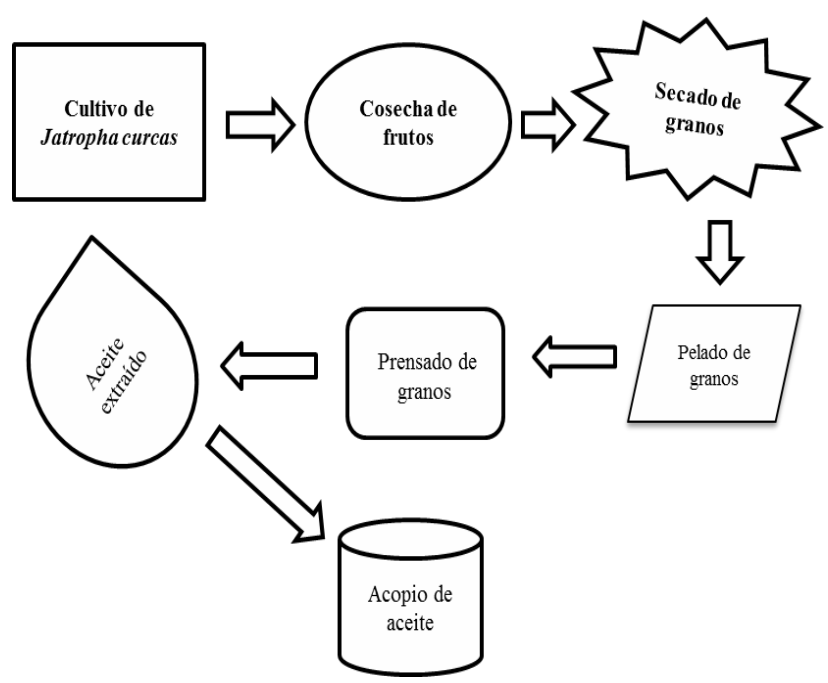

Figura 3. diagrama de obtención del aceite

Se utilizó el Diseño Completo Al Azar, para comparar tres tipos de mezclas con el diésel (tratamientos) con cinco repeticiones, antes de realizar el análisis de varianza se verificó los supuestos tales como: la normalidad de los residuales y homogeneidad de varianzas, utilizando las pruebas de Shapiro Wild y la prueba de Bartlett respectivamente. En los casos que no se cumplieron los supuestos del análisis de varianza se utilizó la prueba no paramétrica de Kruskal Wallis.

\section{Resultados y discusión}

Siguiendo los pasos de la metodología indicada, se realizaron los ensayos en la planta de pruebas (Figura 7 y 8) del Instituto de Motores de Combustión Interna de la Facultad de Ingeniería Mecánica de la Universidad 
Nacional de Ingeniería; habiéndose medido las diferentes variables y luego realizado los cálculos necesarios para llegar a los siguientes resultados:

\section{PRUEBAS DEL DIÉSEL + 0\% DE BIODIESEL.}

Estas pruebas se realizaron con la finalidad de tener como referente y para que sirva como una línea base de las pruebas que luego se debieron realizar con las diferentes mezclas ya indicadas, habiéndose medido y calculado los resultados indicados en las Tablas siguientes:

En la Tabla 1, podemos observar los valores promedios de las pruebas realizadas en la planta de pruebas con el combustible patrón, el Diésel D-2 puro, en él podemos observar que al incrementarse la carga eléctrica, la tendencia de la intensidad de corriente y la opacidad es creciente, éste último sale del límite permitido, por la antigüedad del equipo.

En la Tabla 2, se puede observar los parámetros calculados con los valores medidos de la Tabla 16 en el laboratorio; se calculó la densidad del Diésel D-2, después de haber pesado un litro del diésel, para lo cual se usó una balanza electrónica. A medida que se incrementó la carga eléctrica, la potencia eléctrica se incrementó hasta un máximo de $5.7 \mathrm{KW}$. El consumo específico de combustible fue menor a mayor carga eléctrica; mientras el torque se incrementó a medida que se incrementó la carga eléctrica.

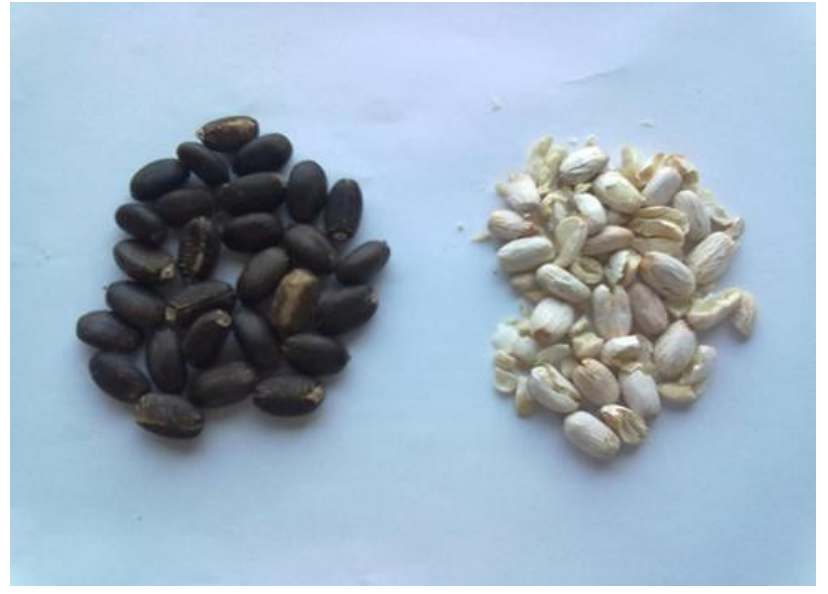

Figura 5. Planta de generación de energía eléctrica

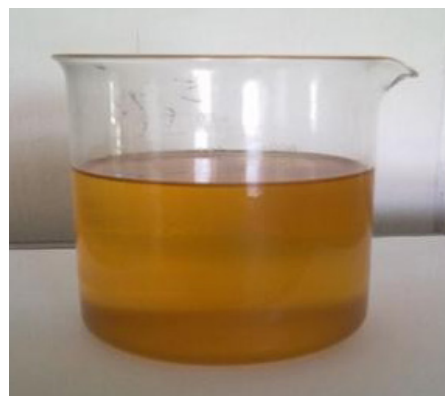

Figura 6. Aceite puro de piñón

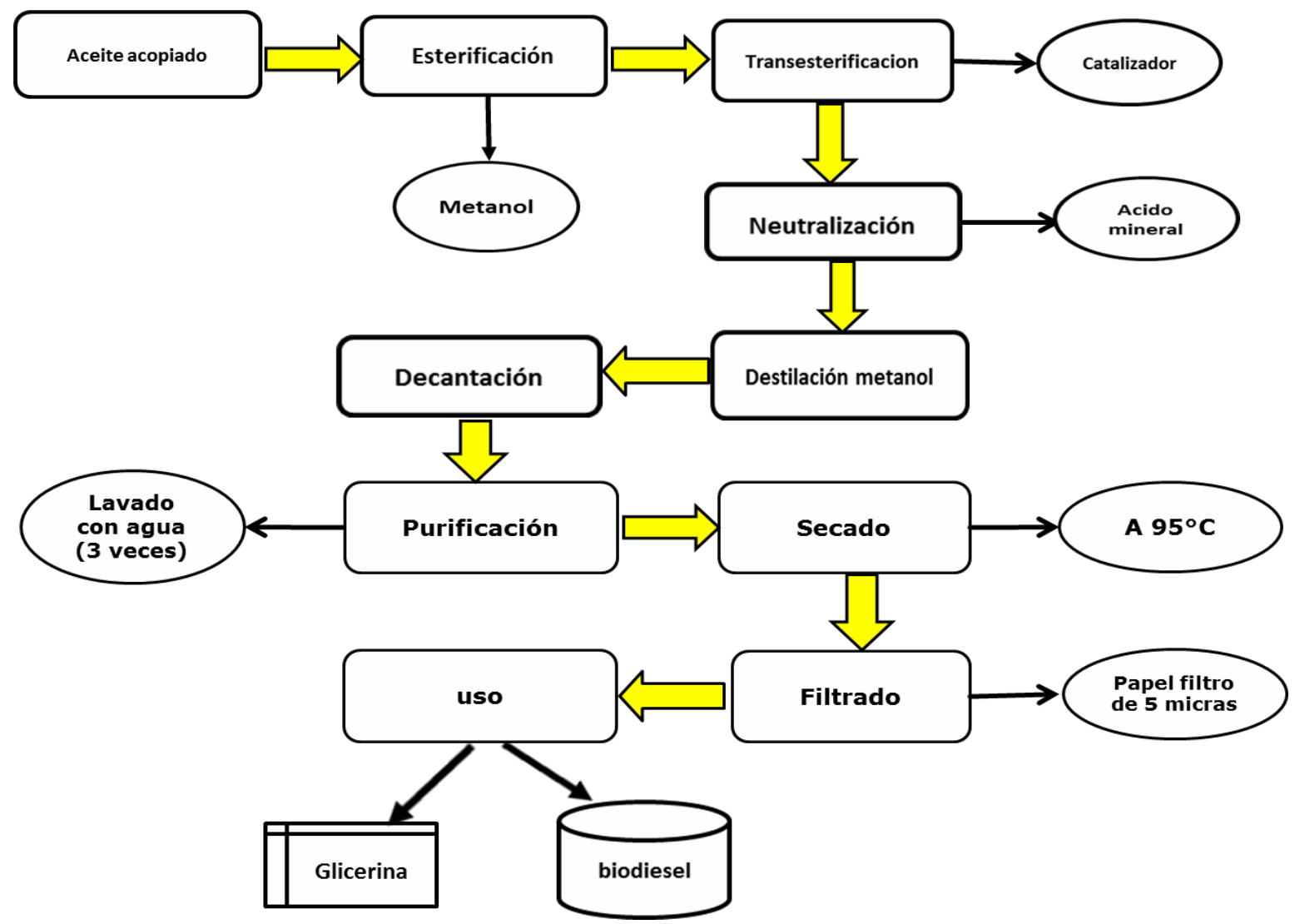

Figura 4. diagrama de obtención del biodiesel de Jatropha curcas 


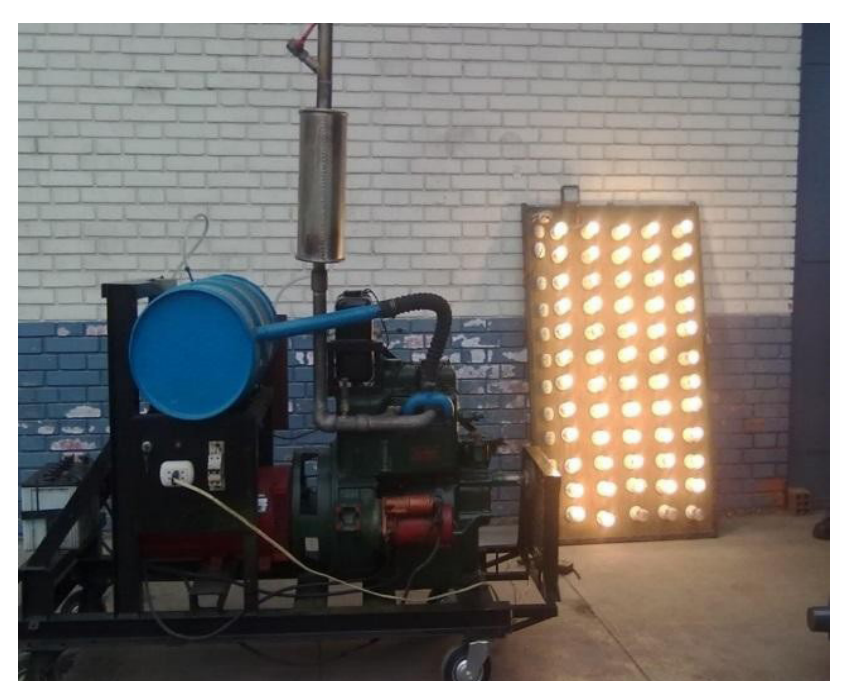

Figura 7. Medición de carga eléctrica plena

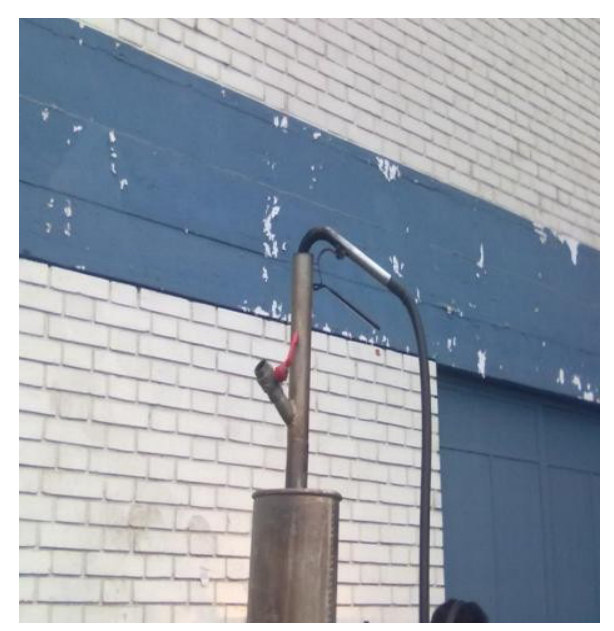

Figura 8. Medición de particulado

Tabla 1. Pruebas con Diésel $+0 \%$ de biodiesel

\begin{tabular}{ccccccccc}
\hline \multicolumn{7}{c}{ Magnitudes medidas } \\
\hline rpm & $\begin{array}{c}\text { Carga } \\
\text { comb. }\end{array}$ & $\begin{array}{c}\Delta \mathrm{V} \\
\text { compo }\end{array}$ & Voltios & amperios & frecuencia & Opacidad \\
$\mathrm{mL}$ & $\mathrm{s}$ & $\mathrm{V}$ & $\mathrm{I}$ & $\mathrm{Hz}$ & $\mathrm{m}$ \\
\hline 1812 & 5 & 5 & 18.02 & 225 & 1.2 & 60 & 1.2 \\
1791 & 10 & 5 & 16.96 & 218 & 2.5 & 60 & 1.47 \\
1819 & 20 & 5 & 14.18 & 222 & 5.4 & 60 & 1.72 \\
1810 & 30 & 5 & 12.97 & 217 & 8.2 & 60 & 1.97 \\
1814 & 40 & 5 & 11.65 & 220 & 10.7 & 60 & 2 \\
1804 & 50 & 5 & 10.58 & 216 & 13.4 & 60 & 1.74 \\
1816 & 60 & 5 & 9.39 & 220 & 16.3 & 60 & 2.5 \\
1813 & 70 & 5 & 8.21 & 218 & 19.1 & 60 & 4.32 \\
1808 & 80 & 5 & 7.38 & 216 & 21.8 & 60 & 6.46 \\
1801 & 85 & 5 & 7.15 & 214 & 23.1 & 60 & 6.15 \\
1797 & 90 & 5 & 6.4 & 213 & 24.2 & 60 & 8.34 \\
1822 & 95 & 5 & 4.91 & 223 & 25.4 & 60 & $>9$ \\
\hline
\end{tabular}

\section{PRUEBAS DEL DIÉSEL D-2 + 10\% DE BIODIESEL}

En la Tabla 3, podemos observar las magnitudes medidas en la planta de pruebas, con el D- $2+10 \%$ de biodiesel en el combustible, y al 95\% de consumo de carga eléctrica, similar al diésel puro, la opacidad superó los $10 \mathrm{~m}^{-1}$. La variación del amperaje también se fue incrementando a medida que aumentamos la carga eléctrica; y el tiempo de consumo de combustible del bulbo se realizó en menor tiempo al incrementarse la carga. Se pudo observar una relación directa entre el incremento de la carga eléctrica y la intensidad de corriente.

En la Tabla 4, podemos observar un ligero incremento de la densidad de la mezcla (D-2 $+10 \%$ biodiesel) con relación al diésel puro, siendo este incremento del $0.86 \%$; así mismo la potencia, el consumo horario de combustible y el torque se incrementó a medida que se iba aumentando la carga eléctrica; mientras que el consumo especifico de combustible fue disminuyendo.

Tabla 2. Parámetros calculados Diésel $+0 \%$ biodiesel

\begin{tabular}{|c|c|c|c|c|}
\hline \multicolumn{5}{|c|}{ Parámetros calculados } \\
\hline Dens. Comb. & $\mathrm{Ne}$ & $\mathrm{Gc}$ & ge & $\mathrm{Me}$ \\
\hline $\mathrm{Kg} / \mathrm{L}$ & $\mathrm{KW}$ & $\mathrm{Kg} / \mathrm{h}$ & $\mathrm{Kg} / \mathrm{KW}-\mathrm{h}$ & $\mathrm{N}-\mathrm{m}$ \\
\hline 0.830 & 0.3 & 0.8 & 2.8 & 1.6 \\
\hline 0.830 & 0.6 & 0.9 & 1.5 & 3.2 \\
\hline 0.830 & 1.2 & 1.1 & 0.9 & 6.3 \\
\hline 0.830 & 1.8 & 1.2 & 0.7 & 9.4 \\
\hline 0.830 & 2.4 & 1.3 & 0.5 & 12.4 \\
\hline 0.830 & 3.0 & 1.4 & 0.5 & 15.9 \\
\hline 0.830 & 3.6 & 1.6 & 0.4 & 18.9 \\
\hline 0.830 & 4.2 & 1.8 & 0.4 & 21.9 \\
\hline 0.830 & 4.7 & 2.0 & 0.4 & 24.9 \\
\hline 0.830 & 5.1 & 2.1 & 0.4 & 27.0 \\
\hline 0.830 & 5.40 & 2.4 & 0.4 & 28.7 \\
\hline 0.830 & 5.7 & 3.1 & 0.5 & 29.7 \\
\hline
\end{tabular}

Tabla 3. Pruebas con Diésel $+10 \%$ de biodiesel

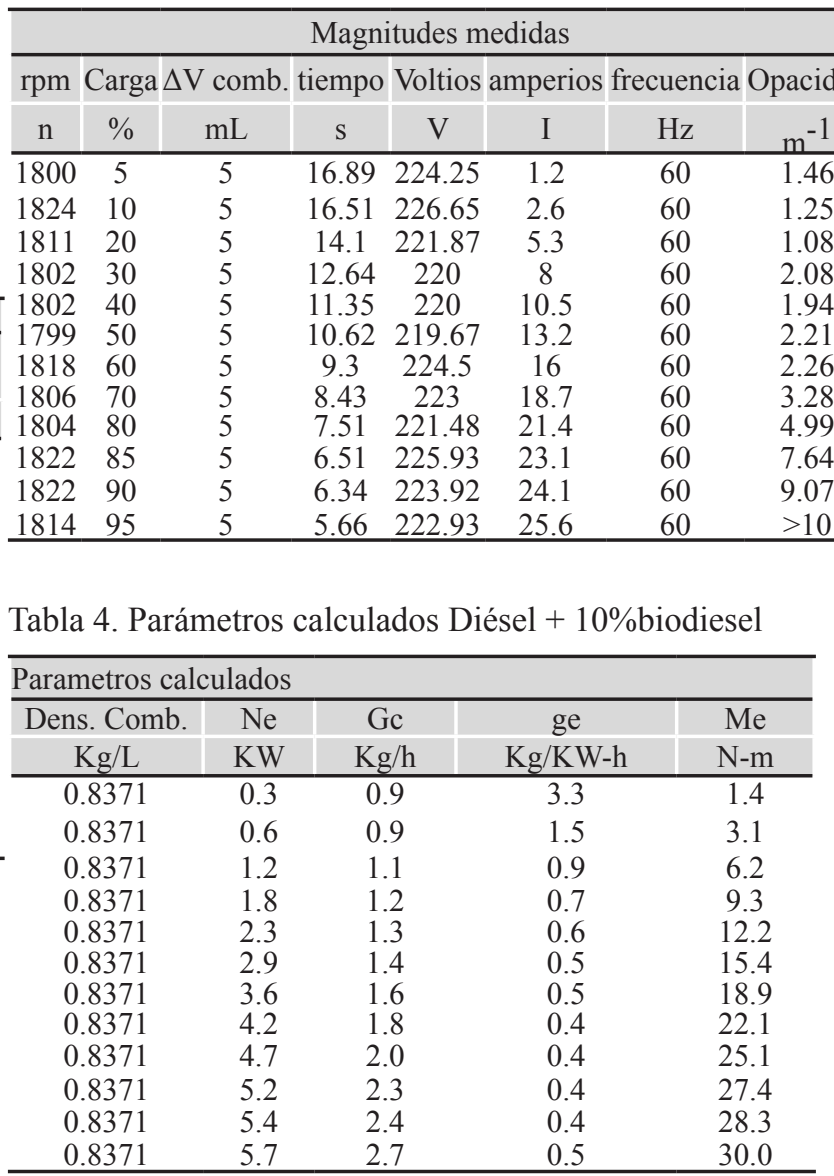


PRUEBAS DEL DIÉSEL D-2 + 20\% DE BIODIESEL

En la Tabla 5, se puede observar que con el $20 \%$ de biodiesel en el Diésel puro el nivel de opacidad registrado fue menor que con el diésel puro y con el 10\% de biodiesel y recién con la carga eléctrica al 100\%, la opacidad superó el nivel $9 \mathrm{~m}^{-1}$.

En la Tabla 6, se puede observar que al ser mezclado el petróleo puro D-2 con $20 \%$ de biodiesel, la densidad se incrementó en $1.23 \%$ y a máxima carga eléctrica la potencia eléctrica se incrementó en $10.53 \%$ respecto al producido con el diésel puro a la misma carga; así mismo el torque también tuvo un incremento del $11.11 \%$ a máxima carga.

Tabla 5. Pruebas con Diésel $+20 \%$ de biodiesel

\begin{tabular}{|c|c|c|c|c|c|c|c|}
\hline \multicolumn{8}{|c|}{ Magnitudes medidas } \\
\hline \multirow{2}{*}{$\begin{array}{c}\mathrm{rpm} \\
\mathrm{n}\end{array}$} & \multirow{2}{*}{$\begin{array}{c}\text { Carga } \\
\% \\
\end{array}$} & \multirow{2}{*}{$\begin{array}{c}\Delta \mathrm{V} \\
\text { comb. } \\
\mathrm{mL}\end{array}$} & \multirow{2}{*}{$\begin{array}{c}\text { tiempo } \\
\mathrm{s} \\
\end{array}$} & \multirow{2}{*}{$\begin{array}{c}\text { Voltios } \\
\text { V }\end{array}$} & \multirow{2}{*}{$\begin{array}{l}\text { amperios } \mathrm{fr} \\
\mathrm{I}\end{array}$} & ecuencia & Opaci \\
\hline & & & & & & $\mathrm{Hz}$ & \\
\hline$\overline{1800}$ & 5 & 5 & 15.19 & 230 & 1.2 & 60 & 1.8 \\
\hline 1805 & 10 & 5 & 13.14 & 227 & 2.6 & 60 & 3.4 \\
\hline 1809 & 20 & 5 & 13.24 & 228 & 5.4 & 60 & 2.0 \\
\hline 1832 & 30 & 5 & 12.23 & 231 & 8.2 & 60 & 1.3 \\
\hline 1836 & 40 & 5 & 11.41 & 230 & 10.8 & 60 & 1.3 \\
\hline 1832 & 50 & 5 & 10.24 & 228 & 13.7 & 60 & 1.6 \\
\hline 1821 & 60 & 5 & 9.37 & 228 & 16.5 & 60 & 1.9 \\
\hline 1808 & 70 & 5 & 8.63 & 226 & 19.2 & 60 & 2.8 \\
\hline 1808 & 80 & 5 & 7.57 & 226 & 22 & 60 & 3.3 \\
\hline 1811 & 85 & 5 & 6.84 & 225 & 23.4 & 60 & 5.9 \\
\hline 1802 & 90 & 5 & 6.57 & 224 & 24.5 & 60 & 5.9 \\
\hline 1830 & 95 & 5 & 6 & 230 & 26.4 & 60 & 7.8 \\
\hline 1821 & 100 & 5 & 5.52 & 228 & 27.6 & 60 & \\
\hline \multicolumn{8}{|c|}{ Tabla 6. Parámetros calculados Diésel $+20 \%$ biodiesel } \\
\hline \multicolumn{8}{|c|}{ Parametros calculados } \\
\hline \multicolumn{3}{|c|}{ Dens. Comb. } & $\mathrm{Ne}$ & Gc & ge & \multicolumn{2}{|r|}{$\mathrm{Me}$} \\
\hline \multicolumn{3}{|c|}{$\mathrm{Kg} / \mathrm{L}$} & KW & $\mathrm{Kg} / \mathrm{h}$ & $\mathrm{Kg} / \mathrm{KW}-\mathrm{h}$ & \multicolumn{2}{|r|}{$\mathrm{N}-\mathrm{m}$} \\
\hline \multicolumn{3}{|c|}{0.8402} & 0.3 & 1.0 & 3.6 & \multicolumn{2}{|r|}{1.5} \\
\hline \multicolumn{3}{|c|}{0.8402} & 0.6 & 1.2 & 2.0 & \multicolumn{2}{|r|}{3.1} \\
\hline \multicolumn{3}{|c|}{0.8402} & 1.2 & 1.1 & 0.9 & \multicolumn{2}{|r|}{6.5} \\
\hline \multicolumn{3}{|c|}{0.8402} & 1.9 & 1.2 & 0.7 & \multicolumn{2}{|r|}{9.9} \\
\hline \multicolumn{3}{|c|}{0.8402} & 2.5 & 1.3 & 0.5 & \multicolumn{2}{|r|}{12.9} \\
\hline \multicolumn{3}{|c|}{0.8402} & 3.1 & 1.5 & 0.5 & \multicolumn{2}{|r|}{16.3} \\
\hline \multicolumn{3}{|c|}{0.8402} & 3.8 & 1.6 & 0.4 & \multicolumn{2}{|r|}{19.7} \\
\hline & 8402 & & 4.3 & 1.8 & 0.4 & & 22.9 \\
\hline & 8402 & & 5.0 & 2.0 & 0.4 & & 26.3 \\
\hline & 8402 & & 5.3 & 2.2 & 0.4 & & 27.8 \\
\hline & 8402 & & 5.5 & 2.3 & 0.4 & & 29.1 \\
\hline & 8402 & & 6.1 & 2.5 & 0.4 & & 31.7 \\
\hline & 8402 & & 6.3 & 2.7 & 0.4 & & 33.0 \\
\hline
\end{tabular}

\section{PRUEBAS DEL DIÉSEL D- 2 + 30\% DE BIODIESEL}

En la Tabla 7, tenemos los valores obtenidos de las pruebas, realizada con la mezcla de $30 \%$ de biodiesel en el D-2 puro, donde se puede observar que también a la máxima carga eléctrica se produjo la mayor opacidad; mientras los demás parámetros medidos fueron muy similares.

En la Tabla 8, podemos observar los parámetros calculados con los datos de la Tabla 7, de la mezcla D-2 con el 30\% de biodiesel, la densidad de la mezcla se incrementó en $1.83 \%$ respecto del petróleo $\mathrm{D}-2$; así mismo podemos observar que el torque se incrementó en $7.74 \%$, respecto del producido a máxima carga eléctrica con el petróleo D-2 puro; así mismo se incrementó la potencia eléctrica en $6 \%$.

En los gráficos que siguen vamos a observar el comportamiento de los diferentes parámetros calculados con las diferentes mezclas en función de la potencia eléctrica desarrollada, tales como:

\section{VARIACIÓN DEL CONSUMO HORARIO DE COMBUSTIBLE}

Calculamos la variación del consumo horario de combustible en función de la potencia eléctrica desarrollada por el motor durante la Generación de energía eléctrica con diferentes mezclas

Tabla 7. Pruebas con Diésel $+30 \%$ de biodiesel

\begin{tabular}{|c|c|c|c|c|c|c|c|}
\hline \multicolumn{8}{|c|}{ Magnitudes medidas } \\
\hline $\mathrm{rpm}$ & Carga & $\Delta \mathrm{V}$ & tiempo & Voltio & amperio & $\mathrm{s}$ frecuencia & Opacidad \\
\hline $\mathrm{n}$ & $\%$ & $\mathrm{~mL}$ & $\mathrm{~s}$ & V & I & $\mathrm{Hz}$ & \\
\hline 1803 & 5 & 5 & 16.39 & 226 & 1.2 & 60 & 1.17 \\
\hline 1804 & 10 & 5 & 15.65 & 227 & 2.6 & 60 & 1.35 \\
\hline 1809 & 20 & 5 & 14.01 & 227 & 5.4 & 60 & 1.75 \\
\hline 1810 & 30 & 5 & 13.13 & 225 & 8.2 & 60 & 1.6 \\
\hline 1808 & 40 & 5 & 11.65 & 225 & 10.8 & 60 & 2.25 \\
\hline 1803 & 50 & 5 & 10.51 & 224 & 13.6 & 60 & 2.74 \\
\hline 1809 & 60 & 5 & 10.9 & 225 & 16.2 & 60 & 3.18 \\
\hline 1802 & 70 & 5 & 8.53 & 226 & 19.1 & 60 & 3.6 \\
\hline 1798 & 80 & 5 & 7.5 & 224 & 21.8 & 60 & 4.2 \\
\hline 1803 & 85 & 5 & 7.31 & 224 & 23.3 & 60 & 6.25 \\
\hline 1813 & 90 & 5 & 6.21 & 226 & 24.7 & 60 & 8.12 \\
\hline 1808 & 95 & 5 & 5.91 & 225 & 26 & 60 & 8.51 \\
\hline 1804 & 100 & 5 & 5.06 & 222 & 27.2 & 59 & $>9$ \\
\hline
\end{tabular}

Tabla 8. Parámetros calculados Diésel $+30 \%$ biodiesel

\begin{tabular}{|c|c|c|c|c|}
\hline \multicolumn{5}{|c}{ Parametros calculados } \\
\hline Dens. Comb. & $\mathrm{Ne}$ & $\mathrm{Gc}$ & ge & $\mathrm{Me}$ \\
\hline $\mathrm{Kg} / \mathrm{l}$ & $\mathrm{KW}$ & $\mathrm{Kg} / \mathrm{h}$ & $\mathrm{Kg} / \mathrm{KW}-\mathrm{h}$ & $\mathrm{N}-\mathrm{m}$ \\
\hline 0.8452 & 0.3 & 0.9 & 3.4 & 1.4 \\
0.8452 & 0.6 & 1.0 & 1.6 & 3.1 \\
0.8452 & 1.2 & 1.1 & 0.9 & 6.5 \\
0.8452 & 1.8 & 1.2 & 0.6 & 9.7 \\
0.8452 & 2.4 & 1.3 & 0.5 & 12.8 \\
0.8452 & 3.0 & 1.4 & 0.5 & 16.1 \\
0.8452 & 3.6 & 1.4 & 0.4 & 19.2 \\
0.8452 & 4.3 & 1.8 & 0.4 & 22.9 \\
0.8452 & 4.9 & 2.0 & 0.4 & 25.9 \\
0.8452 & 5.2 & 2.1 & 0.4 & 27.6 \\
0.8452 & 5.6 & 2.4 & 0.4 & 29.4 \\
0.8452 & 5.9 & 2.6 & 0.4 & 30.9 \\
0.8452 & 6.0 & 3.0 & 0.5 & 32.0 \\
\hline
\end{tabular}

En la Figura 9, podemos observar que el consumo de combustible, se va incrementando casi linealmente hasta los $4 \mathrm{KW}$, pero la tendencia del consumo de combustible fue aparentemente similar a medida que se incrementó la potencia eléctrica.

\section{VARIACIÓN DEL CONSUMO ESPECÍFICO DE COMBUSTIBLE}

Consumo específico, es la cantidad de combustible que necesita un motor para suministrar una determinada unidad 
de potencia por unidad de tiempo. El consumo específico es una forma de expresar el rendimiento del motor, en el sentido de que se relaciona consumo con prestaciones. Cuanto menor sea el consumo específico de un motor, mejor es su rendimiento.

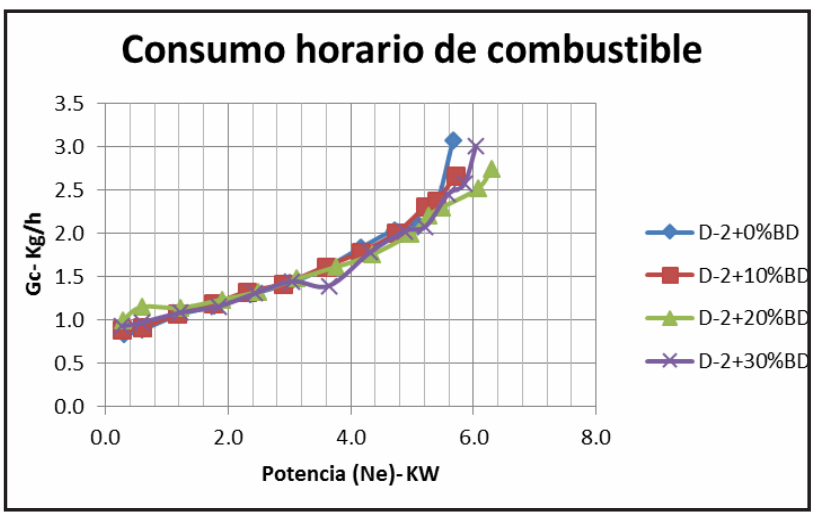

Figura 9. Consumo horario de combustible (elaboración propia)

En la figura 10, podemos observar el comportamiento del consumo especifico de combustible en función de la potencia eléctrica generada, se observó que el consumo específico fue mayor cuando la potencia eléctrica generada fue baja, es decir el rendimiento del motor fue menor, pero a medida que aumenta la potencia eléctrica se observó un comportamiento asintótico, es decir el consumo especifico bajó a $0.5 \mathrm{Kg} / \mathrm{KW}$-h, manteniéndose constante cuando la potencia aumentó, lo que nos indica que en este caso el rendimiento del motor fue alto, manteniéndose constante con cada una de las pruebas, existiendo diferencias significativas entre las mezclas usadas con el $20 \%$ de biodiesel.

Figura 11, se puede observar que el valor de la opacidad generada por el motor de combustión interna de prueba se fue incrementando en los gases del escape a medida que aumentó la potencia eléctrica independientemente de la concentración de biodiesel en el combustible; sin embargo al generase la potencia de $2 \mathrm{KW}$ a $4 \mathrm{KW}$, se pudo observar que el particulado fue menor en la prueba realizada con la mezcla de $30 \%$ de biodiesel, pero al incrementarse la carga y la potencia, la tendencia de la opacidad fue incrementada.

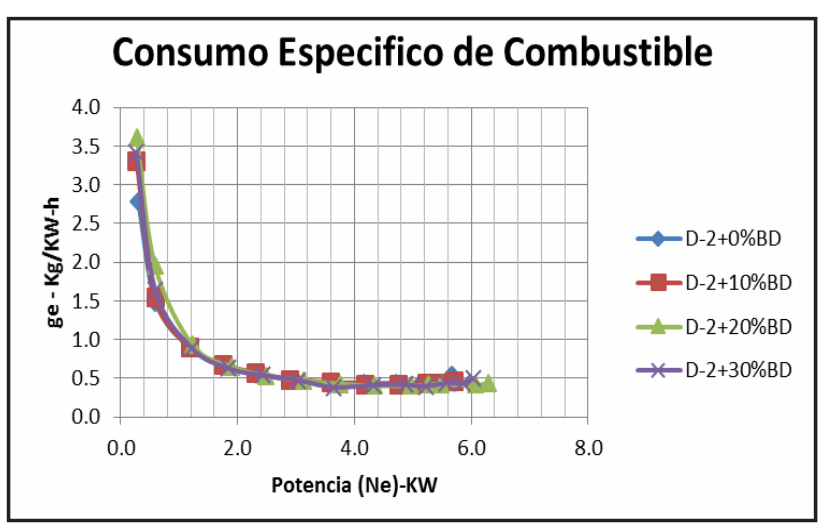

Figura 10. Consumo especifico de combustible

\section{VARIACIÓN DE LA OPACIDAD DE LOS GASES DE ESCAPE}

La opacidad de los gases del escape en función de la potencia eléctrica desarrollada por el motor, con las diferentes mezclas, se puede observar en el grafico siguiente:

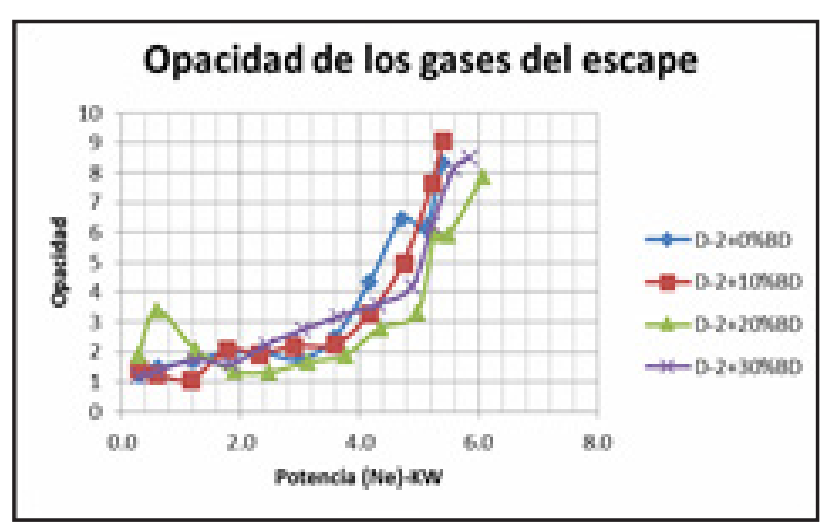

Figura 11. Opacidad medido en el escape de gases del motor

\section{VARIACIÓN DEL MOMENTO DE TORSIÓN DEL MOTOR}

La variación del momento de torsión o torque generado por el motor de combustión interna con diferente carga eléctrica y mezclas en función de la potencia eléctrica desarrollada por el motor, se puede observar en el grafico.

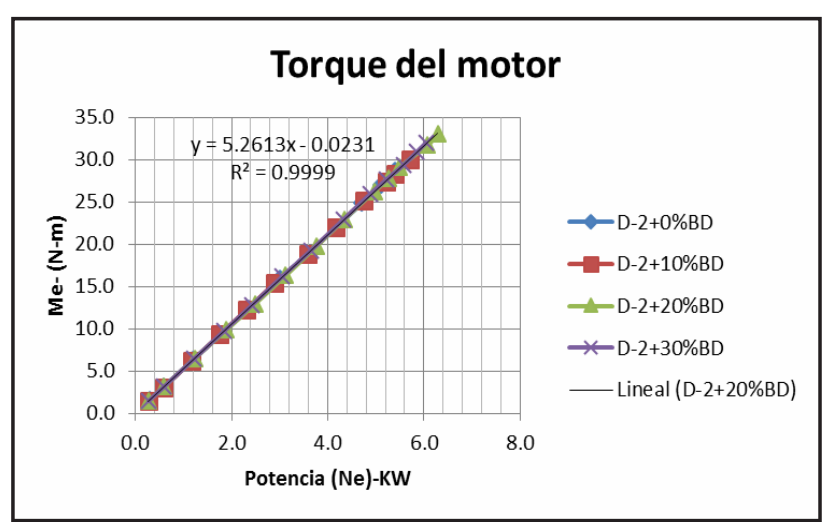

Figura 12. Torque del motor producido con las diferentes mezclas

En la Figura 12, podemos observar que el torque generado en el motor de combustion interna al producirse la potencia electrica a medida que se fue incrementando la carga electrica, tuvo un comportamiento lineal cuya ecuacion se puede observar en el mismo grafico con un $\mathrm{R}^{2}$ del $99 \%$; sin embargo se obtuvo diferencias significativas entre las mezclas; así, las medianas de los valores de torque son mayores con las mezclas del $20 \%$ y $30 \%$; es decir, con estas mezclas el rendimiento del momento torsor fue mayor. 
ANÁLISIS ESTADÍSTICO DE LAS POTENCIAS ELÉCTRICAS GENERADO CON LAS DIFERENTES MEZCLAS

Este análisis se realizó para evaluar los resultados de la potencia eléctrica generada con el diésel puro D-2 y diferentes mezclas, para determinar su nivel de significancia. Esta prueba se calculó con la prueba de Bartlett:

Estadística de prueba $=0.68$, valor $\mathrm{p}=0.877$

\begin{tabular}{lccccc}
\hline $\begin{array}{c}\text { Fuente de } \\
\text { Variación }\end{array}$ & $\begin{array}{c}\text { Grados de } \\
\text { Libertad }\end{array}$ & $\begin{array}{c}\text { Suma de } \\
\text { Cuadrados }\end{array}$ & $\begin{array}{c}\text { Medios } \\
\text { Medrados }\end{array}$ & F & P \\
\hline $\begin{array}{l}\text { Entre Tratos. } \\
\text { (Mezclas) }\end{array}$ & 3 & 0.2880 & 0.0960 & 9.60 & $0.001^{* *}$ \\
$\begin{array}{l}\text { Dentro (error } \\
\text { experimental) }\end{array}$ & 16 & 0.1600 & 0.0100 & & \\
$\begin{array}{l}\text { Total } \\
\mathrm{CV}=4.57 \%\end{array}$ & 19 & 0.4480 & & & \\
\hline
\end{tabular}

El ANVA nos indica que para la fuente de la mezcla (tratamientos) existen diferencias altamente significativas con un coeficiente de variabilidad de $4.57 \%$., es decir de que no todas la potencias promedio son iguales para un $\alpha$ $=0.05$.

Para explorar las diferencias entre las medias de las mezclas se examinó los resultados de las comparaciones múltiples con la prueba de Tukey. (Nos indica que tratamientos resultaron mejor).

\begin{tabular}{lccc}
\hline Mezclas & N & Media & Agrupación \\
\hline D-2+30\%BD & 5 & 3.4800 & A \\
D-2+20\%BD & 5 & 3.4800 & A \\
D-2+10\%BD & 5 & 3.2400 & B \\
D-2+0\% BD & 5 & 3.2400 & B \\
\hline
\end{tabular}

Según la Tabla anterior las mezclas que usaron 20\% y 30\% de biodiesel produjeron similar potencia eléctrica, pero diferentes en relación con la mezcla del $10 \%$ de biodiesel y el diésel D-2 puro.

\section{ANÁLISIS ESTADÍSTICO DEL CONSUMO HORARIO DE COMBUSTIBLE}

El análisis estadístico se realizó con los promedios de los valores de consumo horario de las pruebas realizadas con las diferentes mezclas, y el D-2. Se usó la Prueba No paramétrica de Kruskal-Wallis, que compara las medianas de los promedios, cuyo resultado se observa en el Tabla siguiente:

\begin{tabular}{lrccc}
\hline $\begin{array}{l}\text { Clasificación } \\
\text { Mezclas }\end{array}$ & $\mathrm{N}$ & Mediana & del Promedio & \multicolumn{1}{c}{$\mathrm{Z}$} \\
\hline $\mathrm{D}-2+0 \%$ BD_1 & 5 & 1.650 & 7.0 & -1.53 \\
$\mathrm{D}-2+10 \% \mathrm{BD} \_1$ & 5 & 1.640 & 4.0 & -2.84 \\
$\mathrm{D}-2+20 \% \mathrm{BD} 11$ & 5 & 1.730 & 17.4 & 3.01 \\
D-2+30\%BD_1 & 5 & 1.720 & 13.6 & 1.35 \\
General & 20 & & 10.5 & \\
$\mathrm{CV}=2.58 \%$ & & & & \\
\hline
\end{tabular}

Como se puede observar existen diferencias estadísticas significativas en el consumo horario de combustible entre mezclas, realizado con diferentes cargas eléctricas en el banco de pruebas, en relación a la potencia eléctrica generada, siendo mayores estos con la mezclas de $20 \%$ y $30 \%$ de biodiesel, y menor con la mezcla de $10 \%$ de biodiesel, a un nivel se significación de $\alpha=0.05$.

\section{ANÁLISIS ESTADÍSTICO DECONSUMOESPECÍFICO DE COMBUSTIBLE}

Se utilizó para este análisis la Prueba de Kruskal-Wallis, habiéndose obtenido los siguientes resultados:

\begin{tabular}{lcccc}
\hline $\begin{array}{l}\text { Clasificación } \\
\text { Mezclas }\end{array}$ & $\mathrm{N}$ & Mediana & del Promedio & $\mathrm{Z}$ \\
\hline $\mathrm{D}-2+0 \%$ BD_2 & 5 & 0.8000 & 7.3 & -1.40 \\
$\mathrm{D}-2+10 \%$ BD_2 & 5 & 0.8000 & 7.7 & -1.22 \\
D-2+20\%BD_2 & 5 & 0.9000 & 9.3 & -0.85 \\
D-2+30\%BD_2 & 5 & 0.8000 & 9.0 & -0.65 \\
General & 20 & 10.5 & & \\
CV $=5.52 \%$ & & & & \\
\hline
\end{tabular}

Según los valores anteriores, se puede observar que existen diferencias estadísticamente significativas entre las mezclas, pero el más significativo con la mezcla del $20 \%$ de biodiesel; es decir, el rendimiento del consumo especifico de combustible fue mayor; pero en términos de rendimiento del motor esto significa que con esta mezcla se obtuvo menor rendimiento, mientras que las medianas de los demás fueron prácticamente iguales, es decir, en ellos el rendimiento del motor fue similar.

\section{ANÁLISIS ESTADÍSTICO DE PROMEDIOS DE MOMENTO DE TORSIÓN DEL MOTOR}

Como en los casos anteriores, se usó la Prueba no paramétrica de Kruskal-Wallis para evaluar entre que mezclas se produjo las diferencias de momento Torsión durante los ensayos el uso de las diferentes mezclas.

\begin{tabular}{lcccc}
\hline $\begin{array}{l}\text { Clasificación } \\
\text { Mezcla }\end{array}$ & $\mathrm{N}$ & Mediana & del promedio & $\mathrm{Z}$ \\
\hline D-2+0\% BD_3 & 5 & 16.80 & 7.2 & -1.44 \\
D-2+10\%BD_3 & 5 & 16.68 & 3.8 & -2.92 \\
D-2+20\%BD_3 & 5 & 18.50 & 16.9 & 2.79 \\
D-2+30\%BD_3 & 5 & 18.35 & 14.1 & 1.57 \\
General & 20 & & 10.5 & \\
CV $=5.03 \%$ & & & & \\
\hline
\end{tabular}

De los resultados de la Tabla anterior se puede observar que el torque producido durante las pruebas fue mayor cuando se usó la mezcla con $20 \%$ de biodiesel, seguido con la mezcla del $30 \%$ de biodiesel, pero se obtuvo menor generación del torque con la mezcla de $10 \%$ de biodiesel y el diésel puro D-2. 


\begin{tabular}{|c|c|c|c|c|}
\hline \multicolumn{5}{|c|}{$\begin{array}{l}\text { ANÁLISIS ESTADÍSTICC } \\
\text { OPACIDAD DEL MOTOR }\end{array}$} \\
\hline \multicolumn{5}{|c|}{$\begin{array}{l}\text { Como en los casos anteriores, se usó la Prueba no } \\
\text { paramétrica de Kruskal-Wallis para evaluar entre } \\
\text { que mezclas se produjo las diferencias de emisión de } \\
\text { particulado u opacidad producido durante las pruebas en la } \\
\text { generación de energía eléctrica con el uso de las diferentes } \\
\text { mezclas. }\end{array}$} \\
\hline \multicolumn{5}{|c|}{ Estadística de prueba $=16.24$, valor $\mathrm{p}=0.001$} \\
\hline \multicolumn{5}{|c|}{ Clasificación } \\
\hline Mezcla5 & & Mediana & del promedio & Z \\
\hline$\overline{\mathrm{D}-2+0 \% \text { BD_4 }}$ & 5 & 3.410 & 12.7 & 0.96 \\
\hline D-2+10\%BD_4 & 5 & 3.150 & 3.0 & -3.27 \\
\hline $\mathrm{D}-2+20 \% \mathrm{BD}_{-}^{-} 4$ & 5 & 3.330 & 8.3 & -0.96 \\
\hline D-2+30\%BD_4 & 5 & 3.700 & 18.0 & 3.27 \\
\hline $\begin{array}{l}\text { General } \\
\mathrm{CV}=6.34 \%\end{array}$ & 20 & & 10.5 & \\
\hline
\end{tabular}

Según los resultados de la prueba estadística existen diferencias significativas entre las mezclas sobre los promedios de opacidad de los gases del motor; siendo esto mayor con la mezcla del $30 \%$, eso debido a la antigüedad del motor del banco de ensayo, que para vencer la carga eléctrica completa necesitó mayor esfuerzo, produciendo mayor particulado. La mezcla de $10 \%$ de biodiesel produjo menor particulado.

\section{Conclusiones}

Las pruebas efectuadas con tres concentraciones diferentes de biodiesel $(10 \%, 20 \%$ y $30 \%)$ en el combustible Diesel-2 muestran que los parámetros de potencia y consumo efectivo de combustible del motor difiere significativamente de los resultados obtenidos ensayando el motor con el combustible diésel D-2 puro.

El biodiesel empleado en las concentraciones que van del 10 al $30 \%$ en volumen de mezcla con el combustible Diesel-2 no modifica la tendencia de variación del consumo horario y el consume específico de combustible en función de la variación de la potencia, a velocidad constante.Se obtuvo un significativo aumento de potencia eléctrica cuando el motor funcionó con el $20 \%$ y $30 \%$ de biodiesel, estadísticamente.

En todos los casos, el valor de la opacidad muestra que el motor independientemente de la concentración del biodiesel en el combustible alcanza su potencia nominal con valores de opacidad muy por encima del límite permitido.

Las densidades obtenidas de las diferentes mezclas, se incrementaron levemente respecto al diésel D-2, lo cual le brinda una buena propiedad de lubricidad.

\section{Literatura citada}

Barbosa, et al. 2008. Desempenho comparativo de um motor de gasolina utilizando Ciclo Diesel e mistura de biodiesel. Ciência e Agrotecnologia Lavras., v 32, N5, 1588-1593.
Canturin Chavez, A. 2006. Evaluacion de la Potencia, Torque, Consumo de Combustible y Emision de Particulados empleando Biodiesel de Palma (Elaeis guineensis) puro y en mezclas con petroleo en un motor de Combustion interna. lima-Peru.

Correa, I. 2008. Desempenho de Motor Diesel com misturaas de Biodiesel de Oleo de Girassol. Ciência e Agrotecnologia, Lavras, v.32, n 3, p. 923-928.

Energia, A. M. 2001. Generacion electrica a partir de fuentes nuevas:Energia Solar. Lima.

Ferrari, RA et.al. 2010. Produc ao e uso de biodiesel etilico na UEPG. Obtenido de http://uepg.br/propesp/ publicatio/exa/2004_6/06.pdf

Gonzales Valdes, Roberto. et al. 2010. Consumo de Combustible de los Motores de Combustion Interna. Ciencia Tecnica Agricola, San Jose de las Lajas. v.19 n.1.

Sagarpa. 2009. Programa de Produccion Sustentable de Insumos para Bioenergeticos y de Desarrollo Cientifico y Tecnologico. Mexico: p.18.

Zingg, A. 2004. Evaluacion de la Potencia, torque, consumo de combustible y emision de particulado en un motor empleando petroleo diesel y biodiesel. Lima 